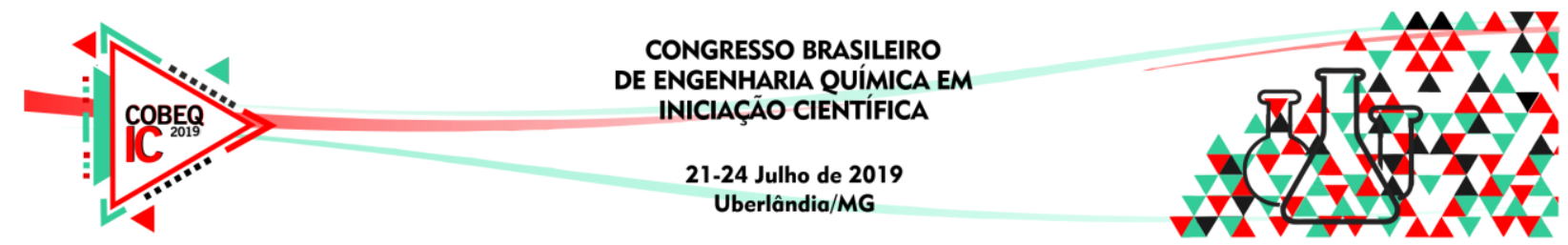

\title{
Estudo da influência dos coeficientes de atrito estático e fricção de rolamento em simulações DEM de tambores rotativos com suspensores
}

\author{
G. F. GRAVENA ${ }^{1}$, J. L. VIEIRA NETO ${ }^{2}$, K. G. SANTOS $^{2}$, B. C. SILVÉRIO ${ }^{1}$ \\ ${ }^{1}$ Universidade Federal do Triângulo Mineiro, Departamento de Engenharia de Alimentos \\ ${ }^{2}$ Universidade Federal do Triângulo Mineiro, Departamento de Engenharia Química \\ *e-mail: beatriz.silverio@uftm.edu.br
}

\begin{abstract}
RESUMO - O Método de Elementos Discretos (DEM), está sendo uma técnica computacional, cada vez mais utilizada, para simular o comportamento de procedimento em fase discreta, e em sistemas particulados. Este trabalho avaliou simulações do escoamento de partículas de fertilizantes super fosfato simples granulado em tambores rotativos, com e sem suspensores. As simulações foram feitas com diferentes combinações de valores de parâmetros no software EDEM ${ }^{\circledR}$, e através da comparação dessas simulações com dados experimentais de massa de sólidos retida nos suspensores foi possível avaliar o efeito de cada parâmetro e da combinação de parâmetros para o escoamento de fertilizantes granulados. Ao todo foram feitas 26 simulações, alterando-se quatro parâmetros: coeficiente de atrito estático partícula-partícula, coeficiente de atrito estático partícula-parede, coeficiente de atrito de rolamento partícula-partícula e coeficiente de atrito de rolamento partícula-parede. Usando uma análise estatística e a técnica de superfícies de resposta para o tratamento dos resultados foi possível verificar a influência de cada parâmetro nos suspensores em nas posições angulares de $0^{\circ}$, $30^{\circ}, 60^{\circ}$ e $90^{\circ}$. Os resultados revelam que nem todos os parâmetros estudados foram significativos. Os parâmetros de atrito estático partícula-partícula e de rolamento partícula-partícula foram significativos na análise. Observou-se que a significância do parâmetro de rolamento partícula-partícula e partícula-parede sofreram alteração com a posição angular.
\end{abstract}

\section{INTRODUÇÃO}

A utilização de tambores rotatórios em indústrias ocorre por ser uma geometria descomplicada e ao seu baixo custo de operação, onde a estrutura é horizontal e cilíndrica que gira em torno do seu próprio eixo, acoplado internamente ao tambor existem suspensores, onde a função é de produzir o cascateamento dos sólidos no interior do secador, assim melhorando o contato com o ar de secagem. Sendo um equipamento excelente para processos como misturas, moagem, secagem de matérias granulados, granulação, fermentação (TJAKRA et al.,2012; SCATENA et al., 2014).

A abordagem Lagrangeana, conhecida também como DEM (Discrete Element Method), consiste no acompanhamento de cada partícula individualmente, juntamente com a conseqüente aplicação do balanço de forças, levando em consideração interações com as demais partículas, esta abordagem se sobressai por permitir a visualização e utilização de partículas irregulares (JUST et al., 2013). Dentre as dificuldades encontradas na abordagem Lagrangeana é possível ressaltar a necessidade de se utilizar coexistências para descrever as 

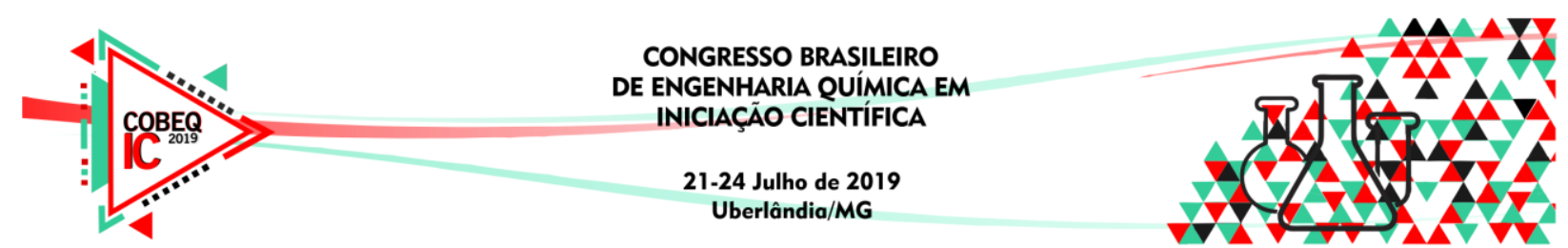

interações partícula-parede, partícula-partícula e partícula-gás, e a dificuldade de prever as variáveis de campo para a fase particulada, dificultando assim a visualização científica dos fenômenos que influenciam as trajetórias das partículas.

Os parâmetros utilizados no projeto e os requisitos operacionais são de extrema importância para a compreensão da interação das partículas dentro de um tambor rotatório. Assim, o objetivo do trabalho é comparar os valores de carga de sólidos nos suspensores em função da posição angular, obtidos através de trabalhos experimentais, com os valores estimados através de simulações DEM, avaliando os diferentes valores dos parâmetros: coeficientes de atrito estático partícula-partícula ( $\mu \mathrm{spp})$, coeficiente de atrito estático partícula-parede $(\mu \mathrm{spw})$, coeficiente de atrito de rolamento partícula-partícula ( $\mu \mathrm{rpp})$ e coeficiente de atrito de rolamento partícula-parede ( $\mu \mathrm{rpw})$.

\section{METODOLOGIA}

Com o intuito de avaliação do efeito dos parâmetros do método DEM sobre o ângulo dinâmico de repouso nos suspensores, bem como a carga de fertilizantes nos supensores, foram realizadas simulações previstas por meio de um planejamento composto central (PCC), no qual a escolha dos valores foi realizada baseando-se em trabalhos da literatura (tabela 1) e assim, realizar a construção da geometria do tambor com 6 suspensores no software EDEM®.

A tabela 1 mostra os valores de coeficientes da literatura.

\begin{tabular}{|l|l|l|l|l|}
\hline & $\boldsymbol{\mu s p p}$ & $\boldsymbol{\mu s p w}$ & $\boldsymbol{\mu r p p}$ & $\boldsymbol{\mu r p w}$ \\
\hline Yiteratura & & & & \\
& 0,6 & 0,6 & 0,01 & 0,05 \\
\hline Marigo et al. (2011) & & & & \\
\hline & 0,5 & 0,35 & 0,01 & 0,005 \\
\hline Hashemia e Spet (2012) & $0,4-2,0$ & $0,4-2,0$ & $0,01-0,09$ & $0,01-0,09$ \\
\hline Ebrahimi e Crapper (2016) & 0,154 & 0,154 & 0,1 & 0,1 \\
\hline Lima (2017) & & & & 0,06 \\
\hline & 0,8 & 0,5 & 0,03 & 0,04 \\
\hline
\end{tabular}

A metodologia de simulação foi baseada nos estudos de Silvério 2012, Lima 2017. Os parâmetros utilizados foram: coeficientes de atrito estático partícula-partícula ( $\mu \mathrm{spp}$ ), coeficiente de atrito estático partícula-parede $(\mu \mathrm{spw})$, coeficiente de atrito de rolamento partícula-partícula ( $\mu \mathrm{rpp}$ ) e coeficiente de atrito de rolamento partícula-parede ( $\mu \mathrm{rpw})$. 


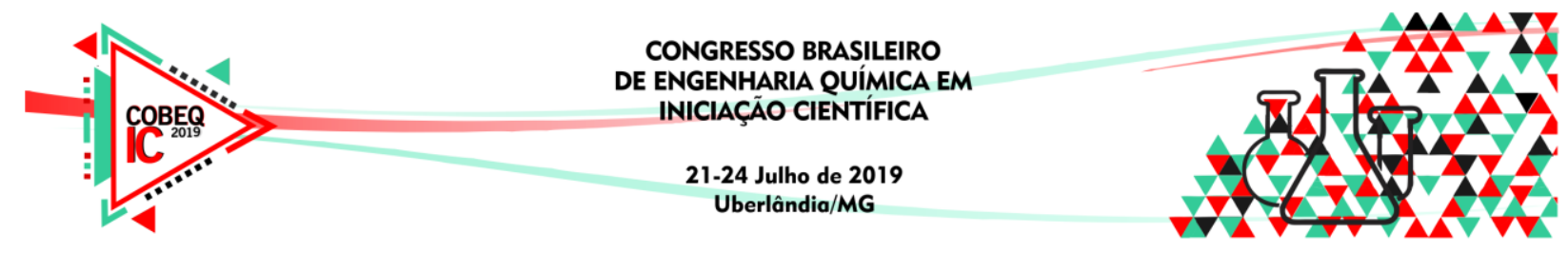

Tabela 2 - Planejamento de simulações com valores dos parâmetros conforme o PCC elaborado.

\begin{tabular}{|c|l|l|l|l|}
\hline Simulações & $\boldsymbol{\mu \text { Spp }}$ & $\boldsymbol{\mu \text { spw }}$ & $\boldsymbol{\mu r p p}$ & $\boldsymbol{\mu r p w}$ \\
\hline 1 & 0.3 & 0.3 & 0.07 & 0.03 \\
\hline 2 & 0.3 & 0.3 & 0.03 & 0.03 \\
\hline 3 & 0.7 & 0.7 & 0.03 & 0.07 \\
\hline 4 & 0.8 & 0.5 & 0.05 & 0.05 \\
\hline 5 & 0.3 & 0.3 & 0.07 & 0.07 \\
\hline 6 & 0.7 & 0.7 & 0.03 & 0.03 \\
\hline 7 & 0.5 & 0.5 & 0.05 & 0.05 \\
\hline 8 & 0.7 & 0.3 & 0.07 & 0.07 \\
\hline 9 & 0.5 & 0.8 & 0.05 & 0.05 \\
\hline 10 & 0.2 & 0.5 & 0.05 & 0.05 \\
\hline 11 & 0.7 & 0.7 & 0.07 & 0.07 \\
\hline 12 & 0.7 & 0.3 & 0.03 & 0.03 \\
\hline 13 & 0.3 & 0.7 & 0.07 & 0.07 \\
\hline 14 & 0.7 & 0.3 & 0.03 & 0.07 \\
\hline 15 & 0.3 & 0.7 & 0.07 & 0.03 \\
\hline 16 & 0.5 & 0.5 & 0.05 & 0.08 \\
\hline 17 & 0.5 & 0.5 & 0.08 & 0.05 \\
\hline 18 & 0.5 & 0.5 & 0.05 & 0.05 \\
\hline 19 & 0.7 & 0.7 & 0.07 & 0.03 \\
\hline 20 & 0.5 & 0.2 & 0.05 & 0.05 \\
\hline 21 & 0.5 & 0.5 & 0.02 & 0.05 \\
\hline 22 & 0.3 & 0.3 & 0.03 & 0.07 \\
\hline 23 & 0.3 & 0.7 & 0.03 & 0.07 \\
\hline 24 & 0.3 & 0.7 & 0.03 & 0.03 \\
\hline 25 & 0.7 & 0.3 & 0.07 & 0.03 \\
\hline 26 & 0.5 & 0.5 & 0.05 & 0.02 \\
\hline & & & & \\
\hline
\end{tabular}

Foram utilizadas as mesmas medidas do tambor rotativo da unidade experimental de forma a se garantir que o tamanho das células fosse no mínimo três vezes maior do que o raio da esfera de igual volume da partícula (dp). Os dados de entrada para a simulação estão contidos na tabela 3 e os não informados foram utilizados os valores sugeridos pelo próprio software. Após a realização da construção da geometria fez-se a injeção das partículas e posteriormente as 26 simulações.

Tabela 3 - Condições para a simulação das metodologias experimentais dos coeficientes de atrito estático e de rolamento no software EDEM.

\begin{tabular}{|ll|}
\hline Modelo & Hertz-Mindlin (noslip) \\
Velocidade Rotacional (rpm) & 3,6 \\
$\mathbf{N}^{\mathbf{0}}$ de particulas & 20583 \\
Tempo simulado $(\mathbf{s})$ & 33 \\
$\mathbf{N}^{\mathbf{0}}$ de suspensores & 6 \\
Diametro do tambor $(\mathbf{m})$ & 0,30 \\
Comprimento do tambor $(\mathbf{m})$ & 0,15 \\
Densidade da particula $\left(\mathbf{k g} / \mathbf{m}^{\mathbf{3}}\right)$ & 1800 \\
Tamanho da célula & $3 \times$ raio da particula \\
\hline
\end{tabular}




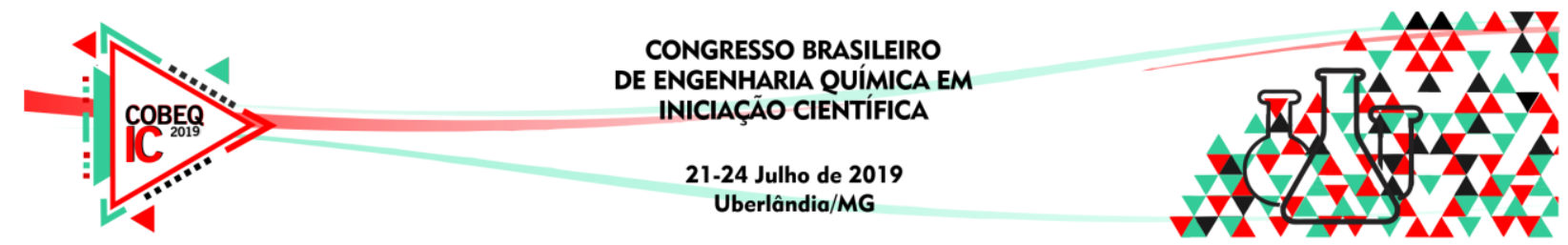

Após realizar as simulações analisou cada uma no tempo de 17 segundos e 18.5 segundos, (após a injeção de partículas) para obter as massas contidas nos suspensores em posições $0^{\circ}, 30^{\circ}, 60^{\circ}, 90^{\circ}$, ou seja, as mesmas posições e tempo avaliados experimentalmente por Silvério 2012.

\section{RESULTADOS E DISCUSSÕES}

Observa-se nas figuras 1 e 2 abaixo que qualitativamente as simulações no EDEM obtiveram resultados compatíveis ao que se vê nas imagens experimentais de Silvério 2012.

Figura 1 - Secador rotativo convencional com os suspensores nas posições $0^{\circ}, 60^{\circ}$ e $120^{\circ}$ : (a) experimento e (b) imagem obtida da simulação 7 do PCC.

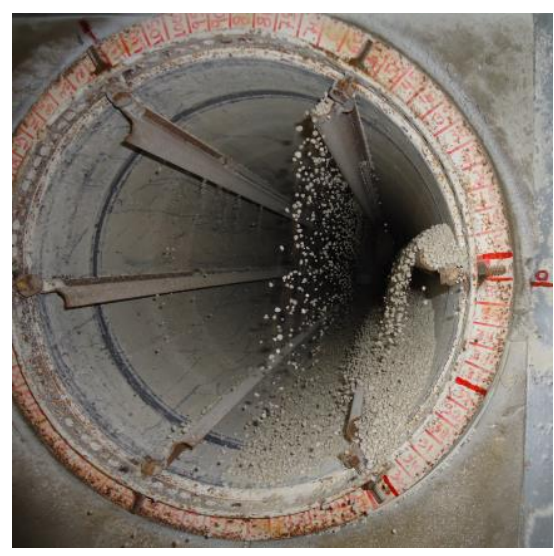

(a) Silvério 2012

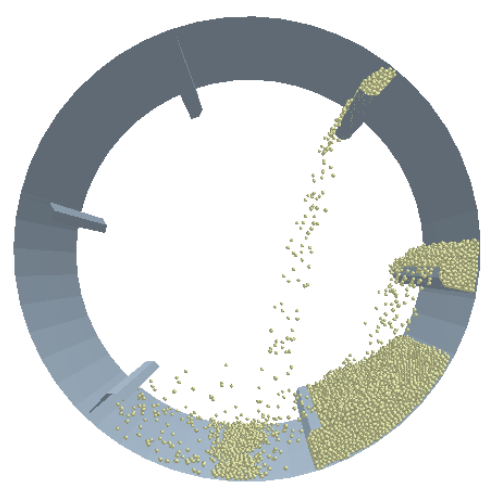

(b) Gravena 2019

Figura 2 - Secador rotativo convencional com os suspensores nas posições $30^{\circ}$ e $90^{\circ}$ : (a) experimento e (b) imagem obtida da simulação 7 do PCC.

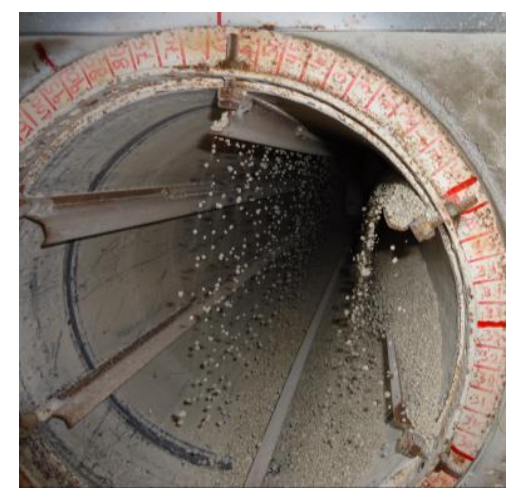

(a) Silvério 2012

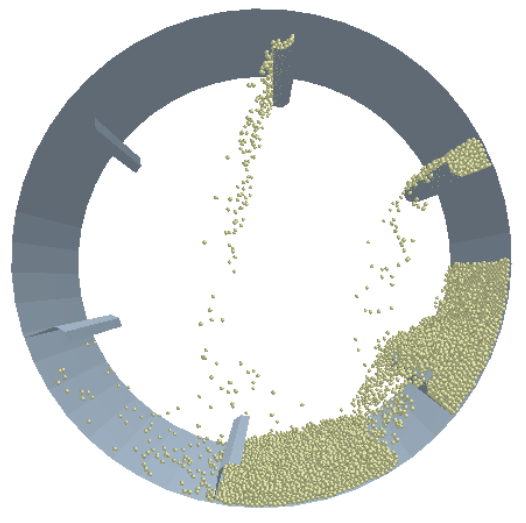

(b) Gravena 2019 
A tabela 4 apresenta os resultados das simulações que foram comparadas com os resultados experimentais de massa de partículas de fertilizante no experimento no tambor rotativo, com a mesma geometria e condições usadas na simulação.

Tabela 4 - Resultados experimentais e das 26 simulações do PCC com diferentes parâmetros de simulação das massas de partículas em $\mathrm{kg}$.

\begin{tabular}{|c|c|c|c|c|}
\hline $\begin{array}{l}\text { Experimento } \\
\text { Silvério } 2012 \\
\end{array}$ & 1,1244 & $\mathbf{0 , 5 9 0 7}$ & 0,4071 & 0,1496 \\
\hline Simulação/ângulo & $\mathbf{0}^{\circ}$ & $30^{\circ}$ & $60^{\circ}$ & $90^{\circ}$ \\
\hline 1 & 1,5031 & 0,9544 & 0,6393 & 0,1567 \\
\hline 2 & 1,4258 & 0,8885 & 0,5576 & 0,1033 \\
\hline 3 & 1,4059 & 0,8942 & 0,5657 & 0,1837 \\
\hline 4 & 1,4812 & 0,9358 & 0,6261 & 0,2111 \\
\hline 5 & 1,5187 & 0,9479 & 0,6470 & 0,1844 \\
\hline 6 & 1,4275 & 0,9108 & 0,5690 & 0,1358 \\
\hline 7 Ponto Central & 1,4741 & 0,9311 & 0,6470 & 0,2067 \\
\hline 8 & 1,5288 & 1,0834 & 0,6629 & 0,3337 \\
\hline 9 & 1,4589 & 0,9425 & 0,6951 & 0,2361 \\
\hline 10 & 1,4427 & 0,9047 & 0,5657 & 0,1378 \\
\hline 11 & 1,5497 & 0,9662 & 0,6450 & 0,0858 \\
\hline 12 & 1,4160 & 0,9105 & 0,5900 & 0,1256 \\
\hline 13 & 1,5106 & 0,9733 & 0,6528 & 0,2151 \\
\hline 14 & 1,4589 & 0,9425 & 0,6177 & 0,2019 \\
\hline 15 & 1,5119 & 0,9571 & 0,6545 & 0,1753 \\
\hline 16 & 1,4900 & 0,9594 & 0,6271 & 0,2101 \\
\hline 17 & 1,5582 & 1,0006 & 0,7007 & 0,2773 \\
\hline 18 Ponto Central & 1,4819 & 0,9311 & 0,6450 & 0,1901 \\
\hline 19 & 1,5339 & 0,9496 & 0,6599 & 0,2438 \\
\hline 20 & 1,4508 & 0,9611 & 0,6194 & 0,1297 \\
\hline 21 & 1,3998 & 0,8736 & 0,5785 & 0,1334 \\
\hline 22 & 1,4136 & 0,8855 & 0,5896 & 0,1388 \\
\hline 23 & 1,4265 & 0,9007 & 0,5684 & 0,1472 \\
\hline 24 & 1,4062 & 0,8929 & 0,5751 & 0,1131 \\
\hline 25 & 1,5173 & 0,9848 & 0,6697 & 0,1915 \\
\hline 26 & 1,4548 & 0,9510 & 0,6170 & 0,1635 \\
\hline
\end{tabular}

Verifica-se que os resultados simulados foram diferentes dos resultados experimentais de Silvério 2012 em todos os ângulos analisados. Assim, a tabela 5 mostra o erro da massa obtida na simulação em relação ao experimento em cada posição angular. 


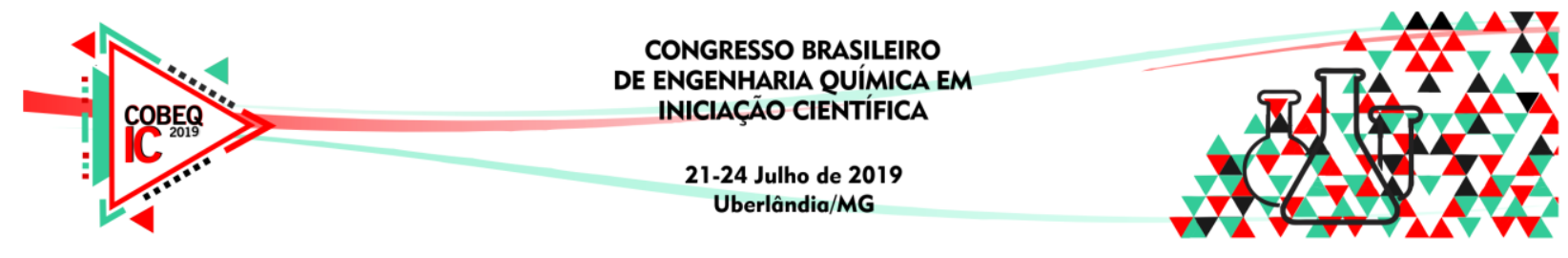

Tabela 5 - Erros percentuais das 26 simulações do PCC com diferentes parâmetros de simulação comparadas com os resultados experimentais de Silvério, 2012.

\begin{tabular}{|c|c|c|c|c|}
\hline Simulação/ângulo & $\mathbf{0}^{\circ}$ & $30^{\circ}$ & $60^{\circ}$ & $90^{\circ}$ \\
\hline 1 & 33,68 & 61,56 & 57,03 & 4,74 \\
\hline 2 & 26,81 & 50,42 & 36,96 & 30,92 \\
\hline 3 & 25,03 & 51,39 & 38,97 & 22,80 \\
\hline 4 & 31,73 & 58,42 & 53,80 & 41,09 \\
\hline 5 & 35,07 & 60,48 & 58,94 & 23,25 \\
\hline 6 & 26,96 & 54,19 & 39,78 & 9,25 \\
\hline 7 & 31,10 & 57,62 & 58,94 & 38,15 \\
\hline 8 & 35,97 & 83,40 & 62,84 & 123,03 \\
\hline 9 & 29,75 & 59,56 & 70,74 & 57,79 \\
\hline 10 & 28,31 & 53,16 & 38,95 & 7,90 \\
\hline 11 & 37,83 & 63,57 & 58,44 & 2,66 \\
\hline 12 & 25,93 & 54,13 & 44,92 & 16,02 \\
\hline 13 & 34,34 & 64,76 & 60,35 & 43,80 \\
\hline 14 & 29,75 & 59,56 & 51,72 & 34,99 \\
\hline 15 & 34,46 & 62,02 & 60,77 & 17,16 \\
\hline 16 & 32,51 & 62,42 & 54,05 & 40,41 \\
\hline 17 & 38,58 & 69,40 & 72,13 & 85,33 \\
\hline 18 & 31,79 & 57,62 & 58,44 & 27,09 \\
\hline 19 & 36,42 & 60,76 & 62,09 & 62,98 \\
\hline 20 & 29,03 & 62,71 & 52,14 & 13,32 \\
\hline 21 & 24,49 & 47,90 & 42,10 & 10,83 \\
\hline 22 & 25,72 & 49,90 & 44,84 & 7,22 \\
\hline 23 & 26,87 & 52,47 & 39,61 & 1,58 \\
\hline 24 & 25,06 & 51,16 & 41,27 & 24,38 \\
\hline 25 & 34,94 & 66,71 & 64,51 & 27,99 \\
\hline 26 & 29,38 & 60,99 & 51,56 & 9,26 \\
\hline
\end{tabular}

Pequenos erros de resultados de massa de partículas na simulação, de até $10 \%$ foram encontrados apenas com o suspensor no ângulo de $90^{\circ}$. Na posição angular de $0^{\circ}$ erros maiores que $25 \%$. A partir desses resultados observa-se que a faixa de parâmetros usada na literatura e apresentada na tabela 1 pode não ser os valores ideais de coeficientes de atrito estático e de rolamento para as condições experimentais aplicadas. Observa-se então a importância de se utilizar os parâmetros corretos para bons resultados de simulação no EDEM $^{\circledR}$ e assim obter maior confiabilidade dos resultados. Assim sendo, verifica-se a 


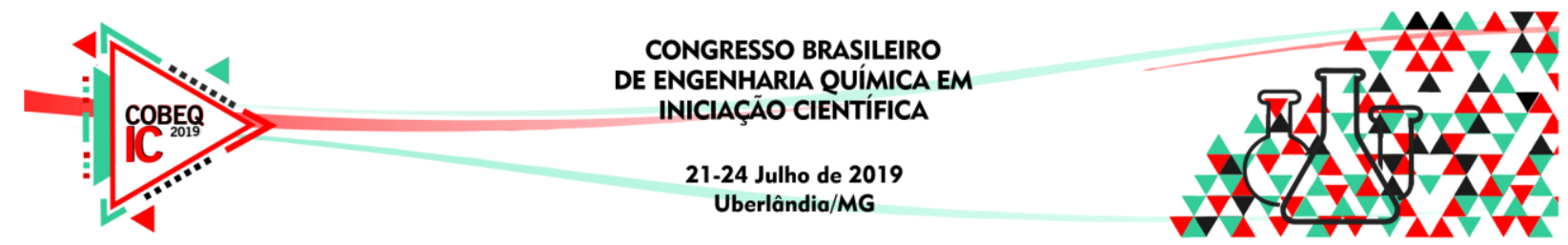

importância de estudos relacionados a estes coeficientes tanto para obtenções experimentais desses parâmetros quanto a verificação e validação dos mesmos na simulação.

Para melhor compreensão da influência dos coeficientes de atrito estático e de rolamento, este trabalho também realizou uma análise estatística dos resultados com a finalidade de observar a significância dos parâmetros nos resultados de massa de partículas em cada posição angular, nas simulações do PCC.

Observou-se na análise estatística do PCC, que a variável linear referente ao coeficiente de atrito estático partícula-partícula, $\boldsymbol{\mu s p p}$, e o coeficiente de rolamento partículapartícula, $\boldsymbol{\mu r p p}$ se mostraram significativos em todas as análises, ou seja para todas as posições angulares. Para as posições angulares de $0^{\circ}$ e $90^{\circ}$ o coeficiente de rolamento partícula parede também se mostrou significativo, o que pode ter ocorrido devido ao maior contato das partículas com a parede do suspensor na posição de $90^{\circ}$, por ter poucas partículas no suspensor e do maior contato das partículas da posição de $0^{\circ}$ com a parede do tambor, durante o movimento de rotação. $\mathrm{Na}$ análise da posição angular de $30^{\circ}$ e de $90^{\circ}$, o coeficiente de atrito estático partícula parede, $\boldsymbol{\mu s p w}$ também se mostrou significativo.

A figura 3 apresenta a variação da massa de partículas na posição angular de $0^{\circ} \mathrm{com}$ o aumento e diminuição dos coeficientes de atrito estático partícula partícula ( $\boldsymbol{\mu s p p}$ ) e de rolamento partícula particula ( $\boldsymbol{\mu r p p})$.

Figura 3 - Superfície de resposta para análise de significância das variáveis ( $\boldsymbol{\mu s p p ) ~ e ~}$ ( $\mu$ rpp).

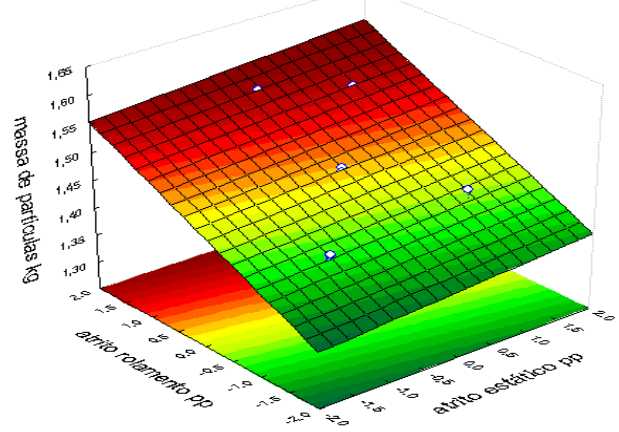

Observa-se efeito mais significativo do $\mu$ rpp quando comparado a influência do $\mu$ spp, para a massa de partículas na posição $0^{\circ}$.

A figura 4 apresenta a variação da massa de partículas na posição angular de $30^{\circ} \mathrm{com}$ o aumento e diminuição dos coeficientes de atrito estático partícula-partícula ( $\boldsymbol{\mu s p p )}$ e de rolamento partícula-partícula ( $\boldsymbol{\mu r p p )}$. 


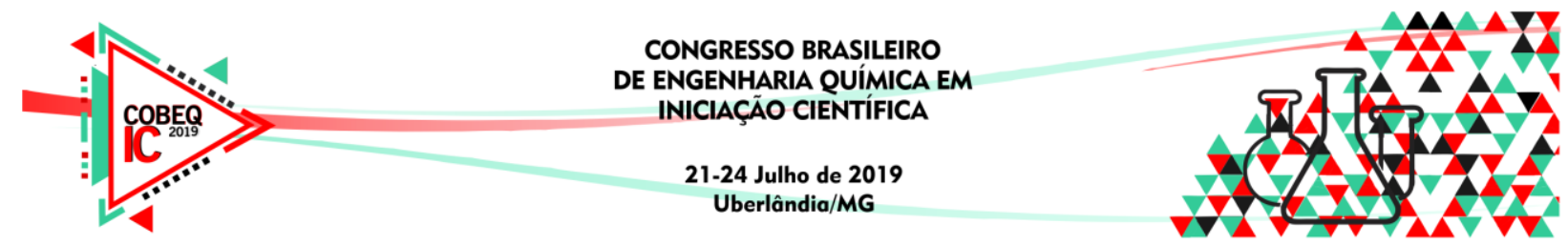

Figura 4 - Superfície de resposta para análise de significância das variáveis ( $\boldsymbol{\mu s p p ) ~ e ~ ( \mu r p p ) . ~}$

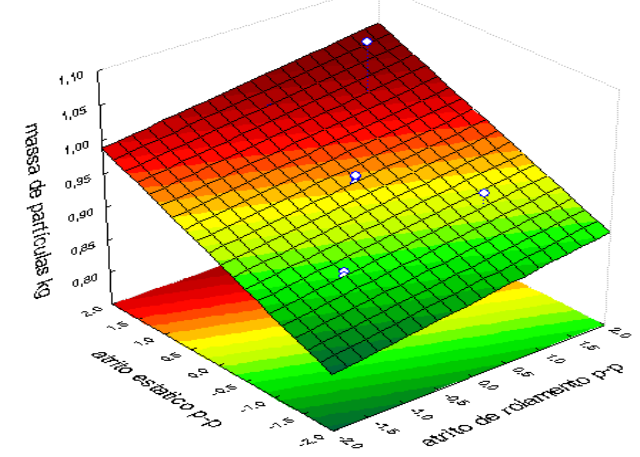

Assim como na posição de $0^{\circ}$ também observa-se efeito mais significativo do $\mu \mathrm{rpp}$ quando comparado a influência do $\mu \mathrm{spp}$, para a massa de partículas na posição $30^{\circ}$.

\section{CONCLUSÕES}

A partir dos resultados acima, pode-se concluir que a faixa de parâmetros escolhida para o estudo não foi a mais adequada para as simulações no software utilizado, sendo necessário um estudo de outras faixas de valores para os materiais deste estudo.

Podem-se observar com os resultados quais foram os parâmetros mais significativos e que os coeficientes de atrito estático e de rolamento partícula-partícula foram os que se mostraram significativos em todos os ângulos analisados, sendo que a influência do coeficiente de atrito de rolamento partícula-partícula apresentou maior influência nos resultados de massa de partículas nos suspensores. 


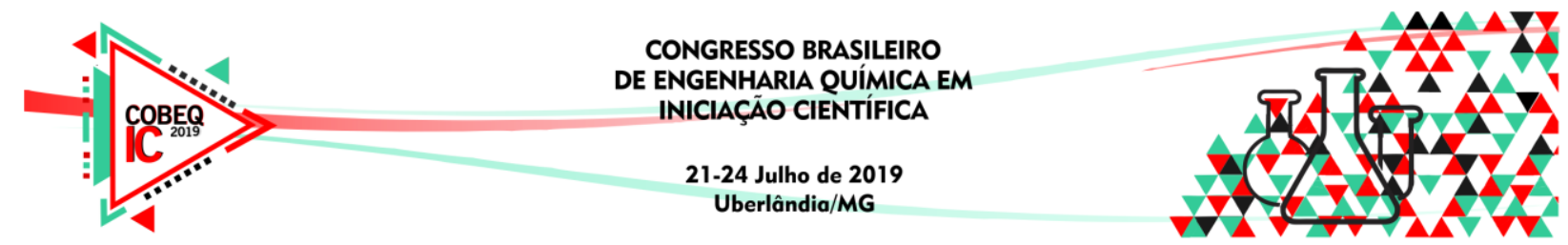

\section{REFERÊNCIAS}

JUST, S.; TOSCHKOFF, G.; FUNKE, A.; DJURIC, D.; SCHARRER, G.; KHINAST, J.; KNOP, K.; KLEINEBUDDE, P. Experimental analysis of tablet properties for discrete element modeling of an active coating process. AAPS PharmSciTech, v. 14, n. 1, p. 402-11, 2013.

LIMA, R. M. Estudo da dinâmica de partículas em tambores rotatórios empregando a abordagem numérica. Dissertação de mestrado. 98 p. PPGEQ-UFU, Uberlândia/MG, 2017.

NASCIMENTO, S. M.. Estudo da Dinâmica de Partículas em tambor rotatório com suspensores: Análise experimental e numérica utilizando abordagens euleriana e lagrangeana. Dissertação de mestrado. 103 p. PPGEQ-UFU, Uberlândia/MG, 2018.

SANTOS, D. A.; DADALTO, F. O.; SCATENA, R.; DUARTE, C. R.; BARROZO, M. A. S. A hydrodynamic analysis of a rotating drum operating in the rolling regime. Chemical Engineering Research and Design, v. 94, p. 204-212, 2015.

SILVÉRIO, B. C.. Estudos fluidodinâmicos e de secagem de fertilizantes em diferentes tipos de secadores rotatórios. 138 p. Tese de Doutorado. PPGEQ-UFU, Uberlândia - MG, 2012. 